\title{
The phenotypic spectrum of proximal $6 q$ deletions based on a large cohort derived from social media and literature reports
}

\author{
Aafke Engwerda ${ }^{1} \cdot$ Barbara Frentz $^{1,2} \cdot$ A. Lya den Ouden ${ }^{1} \cdot$ Boudien C. T. Flapper $^{3} \cdot$ Morris A. Swertz $^{1}$. \\ Erica H. Gerkes ${ }^{1} \cdot$ Mirjam Plantinga ${ }^{1} \cdot$ Trijnie Dijkhuizen $^{1}$ • Conny M. A. van Ravenswaaij-Arts $\mathbb{D}^{1}$
}

Received: 27 September 2017 / Revised: 21 April 2018 / Accepted: 26 April 2018 / Published online: 8 June 2018

(c) The Author(s) 2018. This article is published with open access

\begin{abstract}
Proximal 6q (6q11-q15) deletions are extremely rare and little is known about their phenotypic consequences. Since parents and caregivers now use social media to seek information on rare disorders, the Chromosome 6 Project has successfully collaborated with a Facebook group to collect data on individuals worldwide. Here we describe a cohort of 20 newly identified individuals and 25 literature cases with a proximal $6 \mathrm{q}$ deletion. Microarray results and phenotype data were reported directly by parents via a multilingual online questionnaire. This led to phenotype descriptions for five subregions of proximal $6 \mathrm{q}$ deletions; comparing the subgroups revealed that $6 \mathrm{q} 11 \mathrm{q} 14.1$ deletions presented less severe clinical characteristics than 6q14.2q15 deletions. Gastroesophageal reflux, tracheo/laryngo/bronchomalacia, congenital heart defects, cerebral defects, seizures, and vision and respiratory problems were predominant in those with 6q14.2q15 deletions. Problems related to connective tissue (hypermobility, hernias and foot deformities) were predominantly seen in deletions including the COL12Al gene (6q13). Congenital heart defects could be linked to deletions of MAP3K7 (6q15) or TBX18 (6q14.3). We further discuss the role of ten genes known or assumed to be related to developmental delay and/or autism (BAI3, RIMS1, KCNQ5, HTR1B, PHIP, SYNCRIP, HTRIE, ZNF292, AKIRIN2 and EPHA7). The most influential gene on the neurodevelopmental phenotype seems to be SYNCRIP (6q14.3), while deletions that include more than two of these genes led to more severe developmental delay. We demonstrate that approaching individuals via social media and collecting data directly from parents is a successful strategy, resulting in better information to counsel families.
\end{abstract}

\section{Introduction}

Deletions of the proximal part of the long arm of chromosome 6 , extending from $6 \mathrm{q} 11$ to $6 \mathrm{q} 15$, are rare and information on the related clinical phenotypes is scarce. The largest review to date was published by Hopkin et al. [1] in 1997; it describes 14 individuals with proximal 6q

Electronic supplementary material The online version of this article (https://doi.org/10.1038/s41431-018-0172-9) contains supplementary material, which is available to authorised users.

Conny M. A. van Ravenswaaij-Arts

c.m.a.van.ravenswaaij@umcg.nl

1 Department of Genetics, University of Groningen, University Medical Centre Groningen, Groningen, The Netherlands

2 Vanboeijen, Assen, The Netherlands

3 Department of Paediatrics, University of Groningen, University Medical Centre Groningen, Groningen, The Netherlands deletions, diagnosed by conventional cytogenetic methods. These individuals presented variable degrees of cognitive impairment and minor dysmorphisms: epicanthic folds, short nose with broad nasal tip, anteverted nares, long philtrum and thin upper lip. Pes planus, joint instability, hypermobility, cardiac anomalies, and umbilical and inguinal hernias were also reported. However, the deletions in that study covered different subregions of proximal $6 \mathrm{q}$ and they did not all overlap, therefore the shared clinical features in their cohort may not always be related to the same deleted proximal $6 \mathrm{q}$ segment.

Thanks to the widespread use of microarray techniques, the breakpoint estimation of deletions has become more precise and reproducible, allowing easy and reliable comparison of microarray results from all over the world. In contrast, the objective and precise gathering of phenotypic information is much more complicated. The availability of such information in international databases like ECARUCA (http://www.ecaruca.net) [2] and DECIPHER (https:// decipher.sanger.ac.uk) depends on the time and 
willingness of doctors to submit information. As a result, only a minority of rare cases are being collected and the clinical information in these databases is often incomplete.

We have successfully used social media to gain more knowledge on the 6q11-q15 deletion phenotypes. Patients and parents of children with rare disorders increasingly seek information online and share experiences via patient support groups on social media [3]. Facebook, the largest social network in the world, has a chromosome 6 patient support group, with which we started to collaborate in 2013. Via this group, we were able to collect detailed phenotypic information directly from parents of individuals with a proximal $6 \mathrm{q}$ deletion. Parents and patients were invited to join the Chromosome 6 Project via Facebook and Twitter. The project enabled parents to submit detailed information on their child via an online interactive questionnaire, which was made available in seven different languages. This allowed us to perform genotype-phenotype studies on 20 newly identified individuals, in combination with 25 cases described in the literature. Clinical information and a microarray result were available for all 45 cases. Our approach led to a detailed description of the phenotypic effects of proximal $6 \mathrm{q}$ deletions and to the identification or confirmation of several candidate genes for specific clinical features.

\section{Methods}

Our new cohort was recruited through social media. Since parents provided the clinical information, we have called this our parent cohort. A second group of patients was extracted from the literature (literature cohort).

\section{Parent cohort}

Individuals were informed about the project via Facebook (Chromosome 6 Facebook group), Twitter (@C6study) and our website (https://www.chromosome6.org). Patients or their legal representatives could participate in our research by signing up via our secure website. Inclusion criteria were an isolated chromosome 6 aberration and the availability of a microarray report. Participants received a personal account for the online Chromosome 6 Questionnaire and we obtained their informed consent before they started filling it in. The accredited Medical Ethics Review Committee of the University Medical Centre Groningen waived full ethical evaluation because, according to Dutch guidelines, no ethical approval is necessary if medical information that was already available is used anonymously and no extra tests have to be performed.

Microarray reports were uploaded within the secure environment as part of the sign-up procedure. Genotype data was stored in the Chromosome 6 database and double- checked, after which the original reports were destroyed. The microarray analyses were performed in diagnostic laboratories, using different platforms. The microarray results were converted to GRCh37/hg19 with the UCSC LiftOver Tool and visualised using the UCSC genome browser (http://genome.ucsc.edu). For the present study, we selected participants with a deletion starting in the region $6 \mathrm{q} 11$ to $6 \mathrm{q} 15$ and not extending beyond $6 \mathrm{q} 16$.

Phenotype information was collected via the Chromosome 6 Questionnaire, which was available in English, Dutch, German, French, Italian, Spanish and Portuguese, and constructed with the MOLGENIS toolkit [4]. The questionnaire can only be accessed via a personal account and all answers are automatically stored in a secure environment. The questionnaire contains 132 closed main questions on the pregnancy, birth, congenital abnormalities, relevant dysmorphic features, development, behaviour and health of the child. Questions are only shown when applicable to the participant, based on their age, gender and previously answered questions.

Clinical photographs were collected once written consent was given and were stored on a different server to the database.

Data collected from individuals in the parent cohort was submitted to the ECARUCA database (http://www.ecaruca. net) [2] Id's 5307, 5308 and 5310-5327.

\section{Literature cohort}

Case reports involving proximal $6 \mathrm{q}$ deletions were collected using PubMed and the following search criteria: (deletion or monosomy) and (6q11 or $6 \mathrm{q} 12$ or $6 \mathrm{q} 13$ or $6 \mathrm{q} 14$ or $6 \mathrm{q} 15$ or proximal 6q). Only publications reporting microarray results or comparable detailed breakpoint analysis were included. Clinical information was extracted from the reports using the online Chromosome 6 Questionnaire in order to have as much identical information in the same database as possible.

\section{Data analysis}

All clinical features and behavioural characteristics were classified as present, absent or unknown, and presented as present/known. The developmental delay (intelligent quotient (IQ)) was categorised as normal ( $>85)$, borderline (70-85), mild $(50-70)$, moderate $(30-50)$ or severe $(<30)$ delay. This was based on formal IQ tests or, if these were not available, the mean of the developmental questions for the milestones 'walking independently' and 'using twoword sentences'. The developmental quotients were calculated as the 90th centile of population age of achievement for that milestone divided by the age of achievement in the participant, times 100 . 


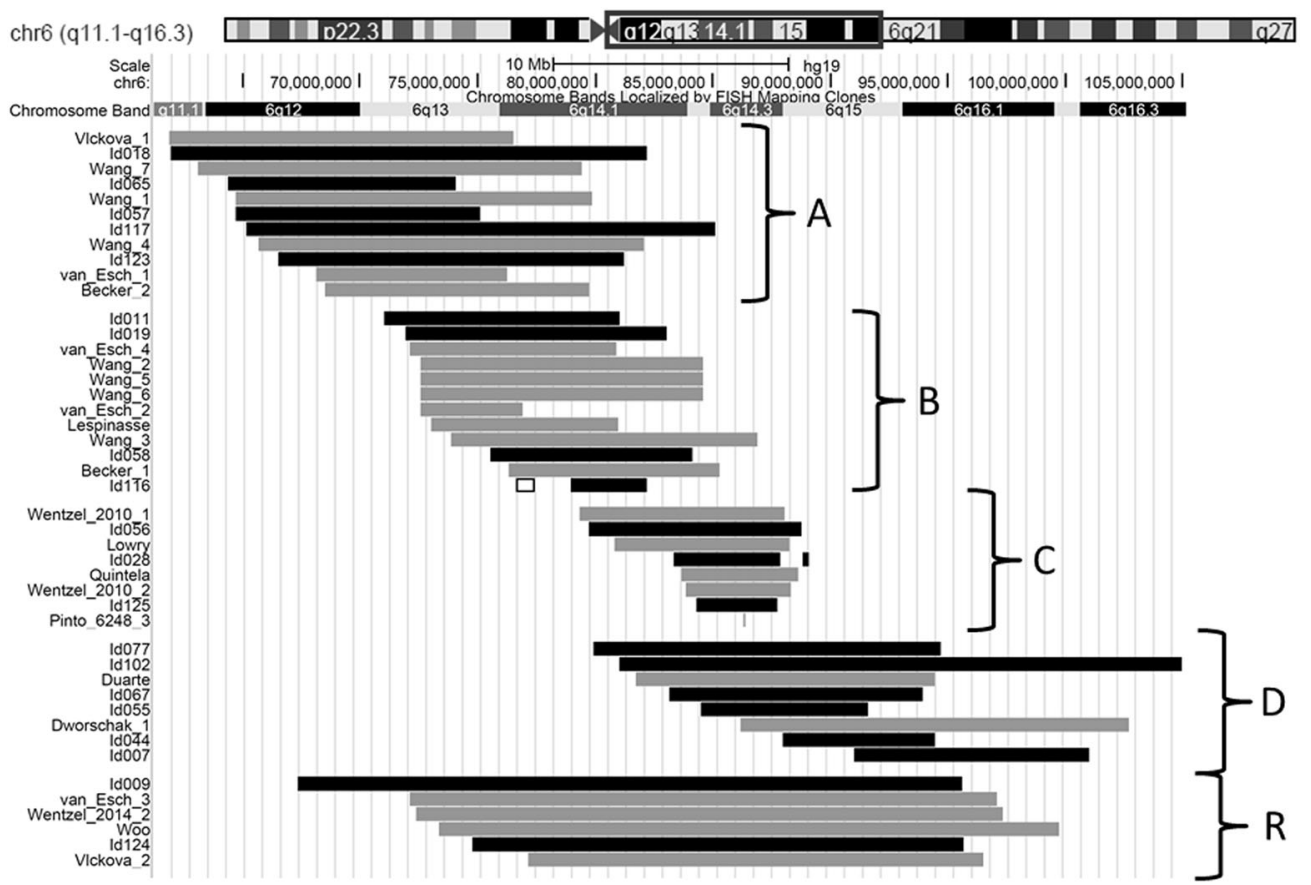

Fig. 1 Overview of all proximal $6 \mathrm{q}$ deletions. Deletions in the region of 6q11-q15 are shown for our parent cohort (black bar) and literature cohort (grey bar), and are divided into five subgroups (A-R). A: deletions of $6 \mathrm{q} 11 \mathrm{q} 13$ extending proximally to $72.5 \mathrm{Mb}$ (calculated from 6pter); B: deletions of $6 \mathrm{q} 13 \mathrm{q} 14.1$ in the region $72.5-84.5 \mathrm{Mb}$; $\mathrm{C}$ : deletions of $6 \mathrm{q} 14.2 \mathrm{q} 14.3$ in the region $81-88.5 \mathrm{Mb}$; and $\mathrm{D}$ : larger deletions of $6 \mathrm{q} 14.2 \mathrm{q} 15$ extending from $81-95 \mathrm{Mb}$. Deletions in

Clinical features were described for the whole group as well as for subgroups, as depicted in Fig. 1-A: deletions of $6 \mathrm{q} 11 \mathrm{q} 13$ extending proximally to $72.5 \mathrm{Mb}$ (calculated from 6pter) $(n=11)$; (B) deletions of $6 \mathrm{q} 13 \mathrm{q} 14.1$ in the region $72.5-84.5 \mathrm{Mb}(n=12)$; (C) deletions of $6 \mathrm{q} 14.2 \mathrm{q} 14.3$ in the region $81-88.5 \mathrm{Mb} \quad(n=8)$; and (D) larger deletions of $6 \mathrm{q} 14.2 \mathrm{q} 15$ extending from $81-95 \mathrm{Mb}(n=8)$. The remaining deletions, subgroup $\mathrm{R}$, were large deletions of 6q12q15 overlapping with two or more of the other subgroups A-D $(n=6)$. The gene content for each subgroup was studied taking into account the haploinsufficiency (HI) and loss-of-function intolerance (pLI) scores. The HI score is defined as the predicted probability that a gene is more likely to exhibit HI (0-10\%), or more likely not to exhibit HI (90-100\%) based on differences in characteristics between known haploinsufficient and haplosufficient genes (https://decipher.sanger.ac.uk) [5]. The pLI score represents the probability that a gene is extremely intolerant of loss-of-function variation (pLI $\geq 0.9$ ). Genes with scores $\leq 0.1$ are loss-of-functiontolerant. This score is based on protein-truncating variants in the ExAC database (http://exac.broadinstitute.org) [6]. We also investigated whether a smallest region of deletion overlap (SRO) could be defined for specific clinical features. subgroup (R) were large deletions of $6 \mathrm{q} 12 \mathrm{q} 15$ overlapping with two or more of the other subgroups A-D. The deletions are visualised using the UCSC genome browser (https://genome.ucsc.edu). Patient Id116 also has a partial duplication of $6 \mathrm{q} 14.1$ (indicated by a white bar). The literature cases were derived from 13 reports [7-19]. See supplementary Table $\mathrm{S} 1$ for details

\section{Results}

\section{Participant characteristics and genotypes}

The online questionnaire was made available in March 2016 and since then 125 parents have registered for an account and uploaded the microarray results of their child. Up to September 2017, 45 parents had completed and submitted the questionnaire. Of these, 20 individuals (13 males and 7 females) had a proximal 6q deletion and could be included in our parent cohort. We further compiled a cohort of 25 literature cases (12 males and 13 females) from 13 published papers [7-19]. The median age (years; months) of individuals in the parent cohort was 5; 5 (range $0 ; 1-24$; 11 ), and in the literature cohort was $5 ; 0$ (range $0 ; 2-22 ; 0$ ).

In all individuals the breakpoints were defined by microarray except for four literature cases [11] in whom the breakpoints were accurately defined by dual-colour FISH analysis using bacterial artificial chromosome clones. Although we excluded individuals with a concomitant aberration of another chromosome, we tolerated some small additional rearrangements based on their size and gene content (see supplementary Table S1 for details and motivation). All individuals were assigned to one of the five subgroups. Figure 1 visualises the deletions, showing the 
Table 1 Genotype characteristics of the deletion 6q11-q15 subgroups

\begin{tabular}{llllll}
\hline Subgroup & $\begin{array}{l}\text { Cohort parent/ } \\
\text { literature }\end{array}$ & $\begin{array}{l}\text { Age median (range) } \\
\text { years; months }\end{array}$ & Size median (range) Mb No. of OMIM genes & $\begin{array}{l}\text { No. of OMIM disease } \\
\text { genes median (range) }\end{array}$ \\
\hline A: q11q13 $(n=11)$ & $5 / 6$ & $3 ; 6(0 ; 1-20 ; 0)$ & $14.71(8.11-20.19)$ & $31(12-49)$ & $11(5-17)$ \\
B: q13q14.1 $(n=12)$ & $4 / 8$ & $5 ; 9(0 ; 9-10 ; 0)$ & $9.48(3.26-13.01)$ & $31(9-47)$ & $11(3-16)$ \\
C: q14.2q14.3 $(n=8)$ & $3 / 5$ & $10 ; 6(2 ; 11-19 ; 0)$ & $4.73(0.02-9.05)$ & $23(2-36)$ & $8(1-11)$ \\
D: q14.2q15 $(n=8)$ & $6 / 2$ & $6 ; 7(0 ; 3-24 ; 11)$ & $11.73(6.43-23.88)$ & $40(19-61)$ & $9(3-14)$ \\
R: q12q15 $(n=6)$ & $2 / 4$ & $1 ; 2(0 ; 2-6 ; 6)$ & $24.95(19.35-28.27)$ & $76(54-80)$ & $20(12-22)$ \\
Total: q11q15 & $20 / 25$ & $5 ; 5(0 ; 1-24 ; 11)$ & $11.19(0.02-28.27)$ & $32(2-80)$ & $11(1-22)$ \\
\hline
\end{tabular}

Genotype characteristics for subgroups A, B, C, D and R, as represented in Fig. 1. The number (median and range) of all OMIM genes and the OMIM disease-related genes within the deletions are given (https://www.omim.org/)

overlap between the different subgroups. The main genotype characteristics of the subgroups are summarised in Table 1 (see supplementary Table S1 for details).

\section{Phenotypes}

Phenotype information is summarised in Table 2 (see supplementary Table S2 for details) and clinical photographs are shown in Fig. 2. Developmental information is given in Table 3 and visualised in Fig. 3 and S1. Common dysmorphic and congenital abnormalities were large size$(36 \%)$ and/or abnormal shape $(44 \%)$ of the head, brain abnormalities (50\%), dysplastic ears $(60 \%)$, cardiac defects (39\%), kidney abnormalities (47\%), abdominal wall hernias (52\%), abnormal genitals in boys $(67 \%)$, vertebral column abnormalities $(41 \%)$ and joint hyperlaxity (53\%). Clinical complications that were most often reported were feeding problems (88\%) and gastroesophageal reflux (55\%), dental problems $(50 \%)$, vision problems $(62 \%)$, recurrent infections (70\%) and hypotonia (76\%). The majority of the individuals was described as being social (60\%) with behavioural problems (68\%) most often reported within the autism spectrum. Developmental delay was seen in all but two participants, child Id057 (subgroup A) and child Id019 (subgroup B), (Fig. S1), who had developed normally when seen at the age of 5 and $3 ; 8$ years, respectively.

Subgroup A (6q11q13) was mostly characterised by the following dysmorphisms and congenital malformations: dysplastic ears (4/8), kidney abnormalities (7/10), abnormal genitals in boys (3/4), umbilical hernia/omphalocele (4/8), syndactyly of the toes $(5 / 8)$ and hypermobility of the joints $(6 / 10)$. Clinical problems that were most frequently encountered were dental problems (3/5), feeding problems $(6 / 7)$ often requiring tube feeding (4/7), recurrent infections (3/5), hypotonia (7/10) and postural problems of the vertebral column (4/7). Developmental delay was mostly mild and most children were described as being social (5/8).
In subgroup B (6q13q14.1), we observed dysplastic outer ears $(6 / 8)$, abnormal genitals in boys $(4 / 5)$, umbilical hernias (7/10), positional foot deformity (5/10) and hypermobility of the joints (5/10). Clinical problems that were most frequently reported were: feeding problems (3/4), short stature (3/8) and hypotonia (8/10). Developmental delay was mostly borderline to moderate. Most children were described as social (5/7), however, some behavioural problems were also reported (3/5).

A distinction was made between subgroups $\mathrm{C}$ (q14.2q14.3) and D (q14.2q15) based on the size of the deletion (Fig. 1). Individuals in subgroups $\mathrm{C}$ and $\mathrm{D}$ had deletions that overlapped. Remarkably, the individuals in subgroup $\mathrm{C}$ were identically effected for most features compared to those in subgroup D, indicating that the region $\mathrm{q} 14.2 \mathrm{q} 14.3$ had the strongest influence on the phenotype. This is exemplified for developmental delay in supplementary Fig. S1, showing that moderate to severe developmental delay is seen in the majority of individuals over age 2 years with a deletion in this region. Subgroups C and D together (6q14.2q15) were mostly characterised by macrocephaly (8/16), brain abnormalities (7/12, especially ventriculomegaly/hydrocephalus and corpus callosum abnormalities), dysplastic outer ears (6/11), congenital heart defects (7/14), kidney abnormalities (4/11) and abnormal genitals in boys (3/7). Medical problems seen in both groups were: vision problems (10/12), gastroesophageal reflux (6/8), constipation (4/8), recurrent infections (7/10), hypotonia (10/ 14) and seizures (5/10). Feeding difficulties (10/11) were frequently seen, but tube feeding was required more often in subgroup D. Epilepsy (3/6) and tracheo/laryngo/bronchomalacia (3/7) was only seen in subgroup D. Most children were described as being social, but behavioural problems were common (6/7), especially autism spectrum disorder, hyperactivity and self-harming behaviour.

As expected, individuals in subgroup $\mathrm{R}$ with larger deletions overlapping the smaller subgroups A-D were more severely affected. The most commonly reported congenital abnormalities were brain abnormalities and 
Table 2 Overview of most prominent characteristics seen in individuals with proximal $6 \mathrm{q}$ deletions

\begin{tabular}{|c|c|c|c|c|c|c|}
\hline Characteristics & Total $(n=45)$ & A $(n=11)$ & $\mathrm{B}(n=12)$ & $\mathrm{C}(n=8)$ & $\mathrm{D}(n=8)$ & $\mathrm{R}(n=6)$ \\
\hline Sex (male/female) & $25 / 20$ & $5 / 6$ & $8 / 4$ & $7 / 1$ & $3 / 5$ & $2 / 4$ \\
\hline Complicated delivery & $18 / 30$ & $3 / 7$ & $5 / 7$ & $6 / 7$ & $3 / 3$ & $1 / 6$ \\
\hline Birth weight $(<\mathrm{p} 10)$ & $9 / 39$ & $0 / 11$ & $2 / 8$ & $2 / 6$ & $2 / 7$ & $3 / 6$ \\
\hline Short stature $(<\mathrm{p} 10)$ & $10 / 36$ & $1 / 9$ & $3 / 8$ & $4 / 8$ & $1 / 6$ & $1 / 5$ \\
\hline Small head circumference $(<\mathrm{p} 10)$ & $3 / 44$ & $3 / 11$ & $0 / 12$ & $0 / 8$ & $0 / 8$ & $0 / 5$ \\
\hline Large head circumference (>p90) & $16 / 44$ & $2 / 11$ & $3 / 12$ & $5 / 8$ & $3 / 8$ & $3 / 5$ \\
\hline Abnormal skull shape & $11 / 25$ & $2 / 6$ & $4 / 7$ & $2 / 4$ & $1 / 5$ & $2 / 3$ \\
\hline Brain abnormalities on MRI or CT & $15 / 30$ & $2 / 6$ & $1 / 6$ & $4 / 5$ & $3 / 7$ & $5 / 6$ \\
\hline Ventriculomegaly/hydrocephaly & $8 / 30$ & $1 / 6$ & $1 / 6$ & $4 / 5$ & $1 / 7$ & $2 / 6$ \\
\hline Vision problems & $16 / 26$ & $2 / 5$ & $1 / 6$ & $6 / 6$ & $4 / 6$ & $3 / 3$ \\
\hline Nystagmus & $7 / 24$ & $0 / 5$ & $1 / 5$ & $2 / 4$ & $1 / 7$ & $3 / 3$ \\
\hline Coloboma & $2 / 24$ & $0 / 5$ & $0 / 5$ & $0 / 5$ & $1 / 6$ & $1 / 3$ \\
\hline Cataract & $3 / 24$ & $0 / 5$ & $0 / 5$ & $2 / 5$ & $0 / 6$ & $1 / 3$ \\
\hline Dysplastic outer ear & $18 / 30$ & $4 / 8$ & $6 / 8$ & $3 / 4$ & $3 / 7$ & $2 / 3$ \\
\hline Mild-moderate hearing impairment & $6 / 25$ & $1 / 5$ & $2 / 5$ & $1 / 6$ & $0 / 6$ & $2 / 3$ \\
\hline Dental problems & $10 / 20$ & $3 / 5$ & $1 / 4$ & $3 / 4$ & $1 / 5$ & $2 / 2$ \\
\hline Feeding difficulties & $23 / 26$ & $6 / 7$ & $3 / 4$ & $5 / 6$ & $5 / 5$ & $4 / 4$ \\
\hline Requiring tube feeding & $12 / 26$ & $4 / 7$ & $1 / 4$ & $1 / 6$ & $3 / 5$ & $3 / 4$ \\
\hline Gastroesophageal reflux & $11 / 20$ & $1 / 5$ & $1 / 4$ & $3 / 3$ & $3 / 5$ & $3 / 3$ \\
\hline Constipation & $9 / 20$ & $2 / 5$ & $2 / 4$ & $2 / 3$ & $2 / 5$ & $1 / 3$ \\
\hline Congenital heart defect & $11 / 28$ & $0 / 5$ & $1 / 5$ & $4 / 6$ & $3 / 8$ & $3 / 4$ \\
\hline Atrial septal defect & $5 / 28$ & $0 / 5$ & $0 / 5$ & $1 / 6$ & $2 / 8$ & $2 / 4$ \\
\hline Tracheo/laryngo/bronchomalacia & $6 / 23$ & $1 / 5$ & $0 / 4$ & $0 / 3$ & $3 / 7$ & $2 / 4$ \\
\hline Recurrent infections & $16 / 23$ & $3 / 5$ & $2 / 4$ & $3 / 4$ & $4 / 6$ & $4 / 4$ \\
\hline Kidney abnormality & $15 / 32$ & $7 / 10$ & $2 / 7$ & $1 / 4$ & $3 / 7$ & $2 / 4$ \\
\hline Abnormal genitals in boys & $12 / 18$ & $3 / 4$ & $4 / 5$ & $3 / 4$ & $0 / 3$ & $2 / 2$ \\
\hline Inguinal or umbilical hernia & $17 / 33$ & $4 / 8$ & $8 / 10$ & $2 / 5$ & $1 / 6$ & $2 / 4$ \\
\hline Vertebral column abnormalities & $11 / 27$ & $4 / 7$ & $1 / 5$ & $4 / 6$ & $0 / 5$ & $2 / 4$ \\
\hline Triphalangeal thumb & $2 / 37$ & $1 / 10$ & $0 / 9$ & $0 / 7$ & $0 / 6$ & $1 / 5$ \\
\hline Pes planus & $6 / 34$ & $3 / 8$ & $1 / 10$ & $1 / 7$ & $1 / 6$ & $0 / 3$ \\
\hline Positional foot deformity & $7 / 34$ & $2 / 8$ & $4 / 10$ & $0 / 7$ & $1 / 6$ & $0 / 3$ \\
\hline Hypermobility of the joints & $18 / 34$ & $6 / 10$ & $5 / 10$ & $0 / 3$ & $1 / 5$ & $6 / 6$ \\
\hline Hypotonia & $28 / 37$ & $7 / 10$ & $8 / 10$ & $5 / 7$ & $5 / 7$ & $3 / 3$ \\
\hline Hypertonia/spasticity & $5 / 24$ & $1 / 7$ & $0 / 4$ & $1 / 4$ & $1 / 6$ & $2 / 3$ \\
\hline Torticollis & $5 / 22$ & $2 / 6$ & $1 / 4$ & $1 / 4$ & $0 / 6$ & $1 / 2$ \\
\hline Seizures/epilepsy & $8 / 27$ & $0 / 7$ & $2 / 8$ & $1 / 4$ & $3 / 6$ & $2 / 2$ \\
\hline Developmental delay (Table 3) & $38 / 40$ & $9 / 10$ & $11 / 12$ & $8 / 8$ & $7 / 7$ & $3 / 3$ \\
\hline Social behaviour & $15 / 25$ & $5 / 8$ & $5 / 7$ & $3 / 4$ & $0 / 4$ & $2 / 2$ \\
\hline Behavioural problems & $19 / 28$ & $3 / 8$ & $3 / 5$ & $7 / 8$ & $5 / 5$ & $1 / 2$ \\
\hline Autism spectrum disorder & $13 / 28$ & $3 / 8$ & $1 / 5$ & $4 / 8$ & $5 / 5$ & $0 / 2$ \\
\hline Hyperactivity & $7 / 28$ & $0 / 8$ & $1 / 5$ & $3 / 8$ & $2 / 5$ & $1 / 2$ \\
\hline Self-harming & $6 / 28$ & $0 / 8$ & $1 / 5$ & $4 / 8$ & $1 / 5$ & $0 / 2$ \\
\hline
\end{tabular}

Clinical features in this table were selected based on their clinical significance and prevalence. For a more detailed overview, see Supplementary table S2

congenital heart defects. Medical problems were low birth weight, feeding difficulties, nystagmus, hearing impairment, recurrent infections, hypotonia, hypermobility and epilepsy.
The severity of developmental delay was only known for two individuals, while behaviour was described as social and hyperactive. 
Fig. 2 Clinical photographs of individuals with a proximal $6 \mathrm{q}$ deletion. Photographs of patients in subgroups A-D. Subgroup A: patient Id123 (A1) at age 22 months, patient Id065 (A2) at age 7 years, patient Id057 (A3) at age 18 months. Subgroup B: patient Id058 (B1) at age 8 years, patient Id019 (B2) at age 7 years, patient Id011 (B3) at age 5 years. Subgroup C: patient Id125 (C1) at age 2; 11 years, patient Id028 (C2) at age 8 years, patient Id056 (C3) at age 2; 5 years. Subgroup D: patient Id007 (D1) at age 8 years, patient Id044 (D2) at age 3 years, patient Id067 (D3) at age 22 years. Written consent was given to the authors to publish the patients' photos
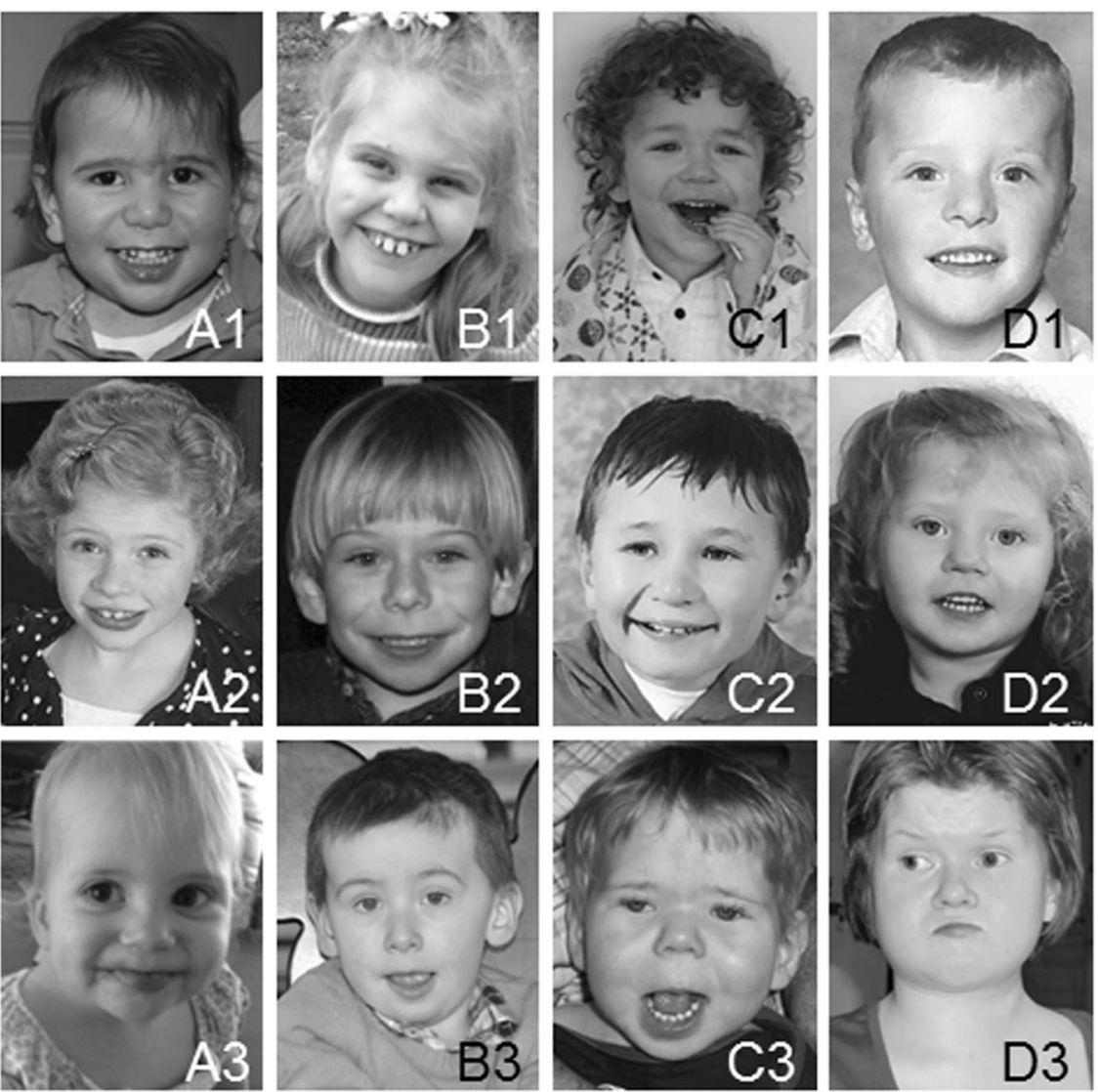

\section{Smallest regions of overlap}

We investigated whether SROs could be defined for the most frequent clinical characteristics listed in Table 2 (data not shown). This was used to construct the phenotype-genotype map (Fig. 4) and to discuss the candidate genes involved (Discussion). Examples are given in supplementary Figs. S2 and S3 for connective tissue-related problems and congenital heart defects, respectively.

Positional foot deformity was present in seven individuals, of whom six had overlapping deletions with an SRO of $4.27 \mathrm{Mb}(72,597-76,869 \mathrm{~kb})$ that includes the candidate gene COL12A1 (collagen type XII alpha 1 chain,

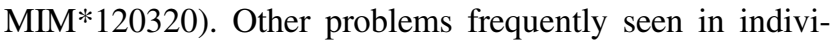
duals with this gene deletion were hernias of the abdominal wall, hyperlaxity and scoliosis (Fig. S2).

Congenital heart defects were seen in 11 patients, of whom six had a deletion of the known cardiac-related gene MAP3K7 (mitogen-activated protein kinase 7, MIM*602614). Four other patients had an overlapping deletion with an SRO of $3.41 \mathrm{Mb}$ (location 84,326-87,736 $\mathrm{kb}$ ) that includes the candidate gene TBX18 (T-box 18, MIM*604613) (Fig. S3). The 11th patient had a deletion breakpoint that was $194 \mathrm{~kb}$ proximal of TBX18.
For the multi-factorial features of developmental delay and autism spectrum disorder, it was difficult to define precisely which part of proximal $6 \mathrm{q}$ contributed most to the phenotype. We therefore depicted the deletions by category (normal/borderline/mild/moderate/severe for developmental delay and present/absent for autism) together with the genes known or discussed to be related to these features (supplementary Figs. S1 and S4). As shown in Table 3, developmental delay was mostly mild to moderate in subgroups $\mathrm{A}$ and $\mathrm{B}$, and moderate to severe in subgroup $\mathrm{C}+\mathrm{D}$. The milestones 'walking independently' and 'using two-word sentences' are visualised in Fig. 3. The severity of developmental delay could not be related to a more specific location or to the length of the deletion (supplementary Fig. S1).

\section{Discussion}

The Chromosome 6 Project aims to achieve a better understanding of the clinical effect of the many different chromosome 6 abnormalities. Here we report our findings for the rare $6 \mathrm{q}$ deletions in the region $6 \mathrm{q} 11$ to $6 \mathrm{q} 15$. The phenotypic effect of a deletion is not the simple addition of the HI effects of the genes within the deleted segment. A 
Table 3 Development for different subgroups of proximal 6q deletions

\begin{tabular}{llllll}
\hline & $\mathrm{A}$ & $\mathrm{B}$ & $\mathrm{C}+\mathrm{D}$ & $\mathrm{R}$ & Total \\
& $\mathrm{q} 11 \mathrm{q} 13$ & $\mathrm{q} 13 \mathrm{q} 14.1$ & $\mathrm{q} 14.2 \mathrm{q} 15$ & $\mathrm{q} 12 \mathrm{q} 15$ & $\mathrm{q11q15}$ \\
\hline Normal & 1 & 1 & 0 & 0 & 2 \\
Borderline & 0 & 2 & 1 & 0 & 3 \\
Mild delay & $\mathbf{6}$ & 1 & 2 & $\mathbf{1}$ & $\mathbf{1 0}$ \\
Moderate delay & 1 & $\mathbf{4}$ & 1 & $\mathbf{1}$ & 7 \\
Severe delay & 1 & 1 & $\mathbf{5}$ & 0 & 7 \\
Delayed but not specified & 1 & 3 & 5 & 1 & 10 \\
Unknown due to young age & 1 & 0 & 2 & 3 & 6 \\
\hline
\end{tabular}

Development categorised as normal (IQ >85), borderline (IQ 70-85), mild (IQ 50-70), moderate (IQ 30-50) or severe (IQ <30) delay. The category with most individuals is highlighted in bold

Not specified $=$ developmental delay is reported, but lacking sufficient information to classify reliably. See also supplementary Fig. S1 deletion of multiple genes may result in a more complex mitigating or reinforcing effect of the under-represented proteins. Moreover, deletion of non-coding stretches of DNA may also affect the phenotype. Thus, it seems best to learn about the clinical effect of deletions by collecting information on individuals with such deletions. Since genetic phenotypes show much inter-individual variation, even when exactly the same gene or chromosome segment is involved, we need information on as many individuals as possible to gain a reliable picture of the effects of a specific chromosome aberration.

Notwithstanding the limitations mentioned above, knowing the gene content of a deletion, especially of genes with a known HI effect, may help to better understand the phenotype. Certain genes may even have direct clinical implications, for example, the deletion of a tumoursuppressor gene leading to an increased risk for cancer and requiring tumour screening. Other genes may be associated with an increased risk for congenital malformations and may thus direct clinical management when known to be deleted. Most genes, however, do not have a known HI effect, or only when they are affected on both alleles, and many small deletions are only considered 'risk factors', ie, are associated with an increased risk for learning problems, intellectual disability or behavioural problems, thereby stressing the multi-factorial nature of the phenotype.

In this discussion, we will relate the clinical phenotypes seen in our cohort to what is already known about the genes involved in the deletions. An overview of the genes within the $6 q 11-6 q 15$ region and with a HI score HI $<50 \%$ (https:// decipher.sanger.ac.uk) is given in Table S3, together with their loss-of-function intolerance score pLI (http://exac.broa dinstitute.org).

\section{Analysis of deletion subgroups}

We collected detailed information on 20 newly identified individuals, who were approached via social media, and studied 25 literature cases. The subgroups showed considerable genetic overlap (especially subgroups A and B), while subgroup $\mathrm{C}$ deletions are smaller but almost fully overlap with subgroup D deletions (Fig. 1). The more proximal subgroups $\mathrm{A}$ and $\mathrm{B}$ presented fewer and less severe clinical characteristics than the more distal subgroups $\mathrm{C}$ and D. The individuals in subgroup R, with the larger deletions, were most severely affected (Table 2). The clinical characteristics of high medical concern, such as gastroesophageal reflux, tracheo/laryngo/bronchomalacia, congenital heart defects, cerebral defects, seizures, and vision and respiratory problems were predominant in the more distal subgroups, $\mathrm{C}$ and D. However, renal problems - mostly hydronephrosis -occurred more often in subgroup A (7/10 individuals), while hypermobility was seen in $6 / 10$ and $5 / 10$ individuals of subgroups $\mathrm{A}$ and $\mathrm{B}$, respectively. Behavioural characteristics, such as autistic behaviour, hyperactivity and self-harming were more often seen in subgroups C and D (12/13) than in subgroups A and B (6/ 13). Most literature reports on deletions of $6 q 14 q 15$ have focused on early-onset obesity, which was not a common problem in our cohort. Cleft palate [10] and syndactyly of the toes [19] have been assigned to $6 q 15$, but were only present in one and two individuals of our cohort, respectively.

The 6q11-q15 region contains 24 genes with a likely clinical effect of $\mathrm{HI}$ based on an $\mathrm{HI}>10 \%$ or a pLI $>0.9$ (supplementary Table S3). Eleven of these likely HI genes have not been associated with a disorder in humans so far: PTP4Al (MIM*601585), PHF3 (MIM*607789), SMAP1 (MIM*611372), EEF1A1 (MIM*130590), SENP6 (MIM*605003), IBTK (MIM*606457), DOPEY1 (MIM*616823), SNAP91 (MIM*607923), CNR1 (MIM*114610), MDN1 (no MIM* available) and $B A C H 2$ (MIM*605394). The other genes are discussed below. 


\section{del 6q11q13 (A)}
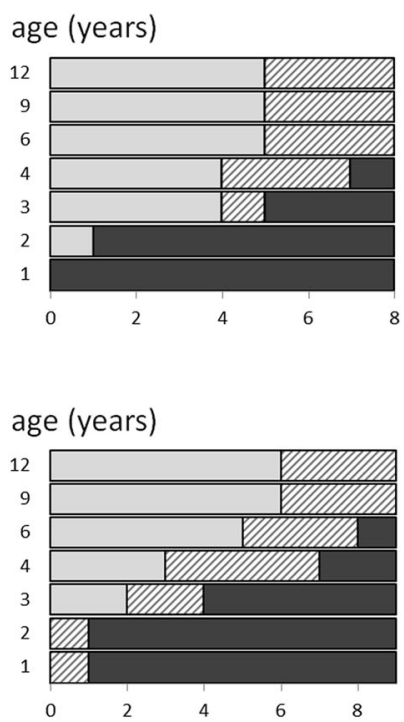

del $6 q 13 q 14.1$ (B) del $6 q 11 q 15$ (Total)

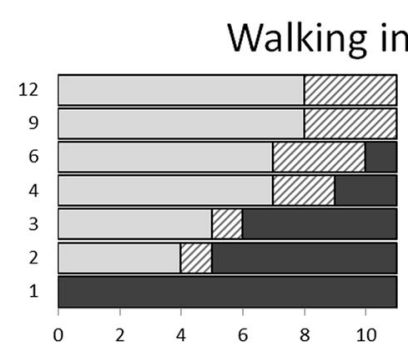

del $6 q 14.2 q 15(C+D)$

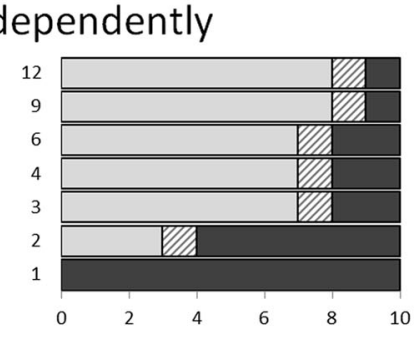

Using two-word sentences

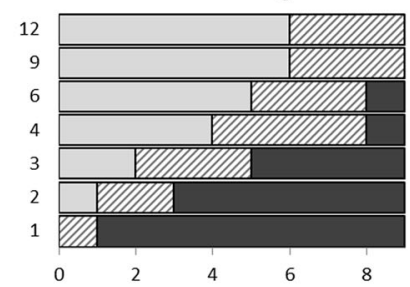

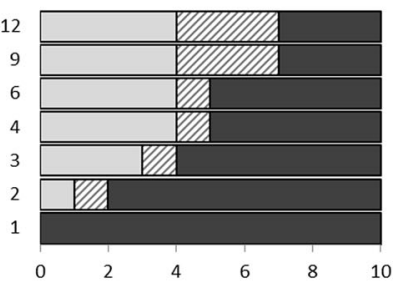

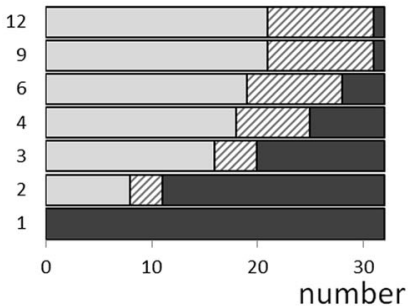

of patients

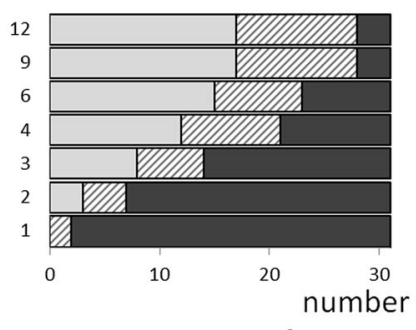

Fig. 3 Age of achievement for the milestones 'walking independently' and 'using two-word sentences'. The light grey bars indicate the number of children ( $x$ axis) that have reached the milestones 'walking independently' (upper panel) and 'using two-word sentences' (lower panel) before the given age ( $y$ axis). The dark grey bars are the children who were not able to perform the milestones at that age. The hatched bars are children who were not able to perform the milestone, but who have not yet reached the age on the $y$ axis. For example, at age 12 years, $66-97 \%$ of the children are able to walk. Only children older than 12 months and for whom information is available were included here.

\section{Developmental delay}

There are six genes located in 6q11-q15 that have been associated with developmental delay (supplementary Fig. S1). BAI3 (adhesion G protein-coupled receptor B3, MIM*602684) was deleted in 12 individuals (11 in subgroup A, 1 in subgroup R) and has been described as a candidate gene for developmental delay by Vlckova et al. [10]. Remarkably, in our cohort, developmental delay varied from none to severe, when this gene was deleted. Lossof-function variants of KCNQ5 (potassium voltage-gated channel subfamily Q member 5, MIM*607357) have been linked to autosomal dominant mental retardation type 46 [20]. This gene was deleted in 24 individuals with no to severe developmental delay (11 in subgroup A, 9 in subgroup B, 4 in subgroup $\mathrm{R}$ ). Three individuals have been described in the literature with most likely loss-of-function variants in PHIP (pleckstrin homology domain interacting protein, MIM*612870) and a comparable phenotype consisting of developmental delay, obesity and dysmorphic features [21, 22]. In our full cohort, $25 / 45$ patients $(6$ in subgroup A, 11 in subgroup B, 2 in subgroup C and 6 in subgroup R) had a PHIP deletion, displayed no to severe developmental delay, and had the following features in common with the three patients with loss-of-function variants: dysplastic ears (10/16), hypotonia (15/19) and

strabism (4/12). Loss-of-function variants in SYNCRIP (synaptotagmin-binding cytoplasmic RNA-interacting protein, MIM*616686) have been detected in individuals with neurodevelopmental disorders and this might explain the more severe developmental delay seen in our subgroup C [23]. SYNCRIP was deleted in 20 individuals who almost all had moderate to severe delay ( 1 in subgroup B, 7 in subgroup C, 6 in subgroup D, 6 in subgroup R). AKIRIN2 (akirin 2, MIM*615165) has been shown to be essential for cerebral cortex development in knockout mice [24]. No phenotype is known in humans but six individuals in our cohort have a deletion of this gene and moderate to severe developmental delay. EPHA7 (ephrin receptor A7, $\mathrm{MIM}^{*} 602190$ ) is known to be expressed in the brain [25], but there is no firm evidence that it is related to a neurodevelopmental disorder and we do not see a more severe developmental phenotype in individuals who lack SYNCRIP, AKIRIN2 and EPHA7 (subgroups D and R) compared to those who only lack SYNCRIP and AKIRIN2 (subgroup C). As shown in Fig. 4 and S1, the most important region for severe developmental delay seems to be 6q14.2q14.3 (subgroup C) and the most important genes involved are SYNCRIP and AKIRIN2. However, the other genes known to be related to developmental delay-BAI3, KCNQ5, PHIP and EPHA7-may also have an effect. 


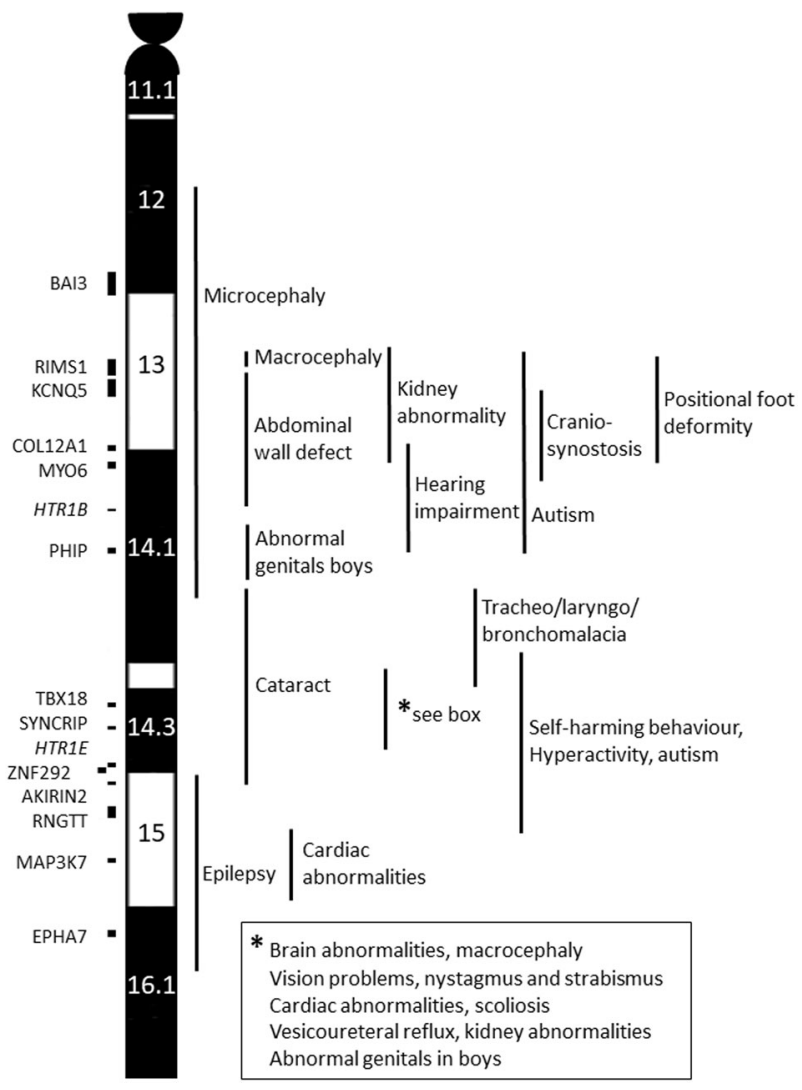

Fig. 4 Phenotype-genotype map of proximal chromosome 6q. An idiogram of proximal $6 \mathrm{q}$ is shown: genes with a known or likely phenotypic effect and an HI score $<10 \%$ or a pLI score $>0.9$ are shown on the left. Genes printed in italics have a higher HI\% or lower pLI, but are discussed in the text as being related to autism. On the righthand side, the critical regions for various clinical features are indicated

As shown in Fig. S1, deletion of only PHIP or only SYNCRIP is enough to cause severe developmental delay, but most individuals with deletions of these genes show borderline to severe, or mild to severe developmental delay, respectively. On the other hand, all those with a deletion of at least three of the six genes related to developmental delay have mild to severe delay, so there is an indirect effect from deletion size.

\section{Autism}

Autism spectrum disorders are frequently described in chromosomal syndromes and an increasing number of genes have been associated to this behavioural phenotype. Autism-related behaviour was predominantly seen in subgroups C and D (Table 2 and supplementary Fig. S4). Genes located in proximal $6 \mathrm{q}$ that have been linked to autism are RIMS1 (regulating synaptic membrane exocytosis 1, MIM*606629), PHIP, SYNCRIP and ZNF292 (zinc finger protein $292, M^{*} M^{*} 616213$ ). A missense mutation in RIMS1 has been identified in one family with cone-rod dystrophy type 7 , with an age of onset from 14 to 42 years
[26]. This eye disorder was not observed in our cohort. However, others have shown an increased number of truncating RIMS1 variants in individuals with autism spectrum disorder [27, 28]. Thus, deletion of RIMS1 might be a risk factor for autism. In our cohort, 22 individuals (11 in subgroup A, 8 in subgroup B, 3 in subgroup R) had a complete or partial deletion of RIMS1. Information on behaviour was available for 13 of them but only three had autism-like behaviour. PHIP variants have been identified in individuals with autism [29]. However, in our cohort, only $3 / 14$ individuals with a PHIP deletion had an autism spectrum disorder. In the meta-analysis by Lelieveld et al. [23], SYNCRIP was identified as a gene for intellectual disability and Pinto et al. [18] described one patient with a small de novo deletion of only $23 \mathrm{~kb}$ including part of the SYNCRIP gene (see Pinto_6248_3 in Fig. 1 and S4). This patient had severe developmental delay and autism, indicating that autism may be part of the SYNCRIP-related phenotype. In total, 7/13 patients with a SYNCRIP deletion in our cohort had an autism spectrum disorder. There is one report of likely disruptive variants in ZNF292 being associated with autism [29] and we observed autism in 4/10 individuals with a ZNF292 deletion. More studies are needed to draw definite conclusions on the role of this gene in autism.

Apart from these four known genes, the genes HTRIE (5hydroxytryptamine receptor $1 \mathrm{E}, \mathrm{MIM}^{*} 182132$ ) and $H T R 1 B$ (5-hydroxytryptamine receptor 1B MIM*182131), which did not pass our strict HI score, may also play a role in autism-like behaviour (supplementary Table S3 and Fig. S4). These genes both code for serotonin receptors. Serotonin is a neurotransmitter that plays a role in various cognitive and behavioural functions, including feeding, sleep, pain, depression and learning [30]. A case-control study in 252 individuals with autism spectrum disorder suggested a role for $H T R l B$ in the predisposition to autism, since such individuals more often had polymorphic HTRIB variants that were known to have a lower expression [31]. Remarkably, we observed an autism spectrum disorder in 7/13 individuals with an HTRIE and a SYNCRIP deletion, while the numbers were $3 / 13$ for RIMS1, 3/12 for HTR1B, 3/14 for PHIP and 4/10 for ZNF929 (supplementary Fig. S4).

\section{Connective tissue-related problems}

Hypermobility, foot deformities and hernias were more often seen in subgroups A and B. COL12Al is thought to be responsible for these connective tissue-related problems in the most proximal $6 \mathrm{q}$ deletions. Other disorders linked to this gene are due to recessive or dominant-negative mutations (Ullrich congenital muscular dystrophy 2 (MIM\#616470) and Bethlem myopathy 2 (MIM\#616471)). If we analyse the clinical features of all the individuals in 
groups A, B and R with a COL12A1 deletion $(n=24)$, we see hernias in $13 / 18$, hyperlaxity in $15 / 21$, foot deformities in $8 / 16$ and kyphosis/scoliosis in 7/12, thereby supporting the role of this gene in the connective tissue phenotype. In supplementary Fig. S2, we show that 14/16 individuals who have at least two of the above features, have a deletion of COL12A1. Another collagen gene in this region is COL9A1 (collagen type IX alpha 1 chain, MIM*120210), which is associated with cartilage problems. However, this gene has an $\mathrm{HI}$ of $24 \%$ and a pLI of 0 , indicating that a $\mathrm{HI}$ effect is very unlikely.

\section{Deafness}

MYO6 (myosin VI, MIM*600970) is involved in autosomal dominant deafness type 22 (DFNA22) due to both missense and truncating mutations [19] and thus might explain the deafness seen in 5/23 individuals with a deletion including MYO6. However, in DFNA22, deafness is progressive with an age of onset of 20 years and onwards, while the individuals with hearing problems in our cohort range in age from 8 months to 22 years, with an unknown age of onset of their hearing loss.

\section{Heart defects}

Congenital heart defects were present in 11 patients, of whom six had a deletion of MAP3K7, a gene known to be related to cardiac defects, while nine had a deletion of our candidate gene TBX18 (supplementary Fig. S3). Together they covered the deletions of 10/11 individuals with a heart defect. The single patient who had neither a deletion of MAP3K7 nor TBX18 had a breakpoint $194 \mathrm{~kb}$ proximal to $T B X 18$, so a position effect cannot be excluded. MAP $3 K 7$ is a well-known disease gene, with missense or non-truncating mutations resulting in autosomal dominant frontometaphyseal dysplasia type 2 (MIM\#617137) and cardiospondylocarpofacial (MIM\#157800) syndromes, while deletions seem to have a different phenotypic effect [32]. Nonetheless, heart defects are part of the cardiospondylocarpofacial syndrome and we observed heart defects in $6 / 12$ individuals with a MAP $3 K 7$ deletion. TBX18 is thought to be responsible for the development of the myocytes in the ventricular septum and the atrial and ventricular walls of the heart although cardiac disease-related variants in the human TBX18 gene have not been reported. However, heterozygous variants within the TBX18 gene promoter were reported in 4 out of 326 individuals with a ventricular septal defect, while no functional variants were found in a control group $(n=327)$ [33]. Most individuals with a heart defect in our cohort presented with an atrial septal defect. TBX18 is also known to be associated to congenital kidney and urinary tract anomalies. In our total cohort $(\mathrm{C}, \mathrm{D}, \mathrm{R}), 19$ individuals had a TBX18 deletion, of whom $6 / 13$ had a kidney abnormality and 9/15 had a congenital heart defect.

\section{Limitations to our findings}

Our subgroups are very small due to the rarity of chromosome 6 aberrations. We had detailed information for 20 participants from our Chromosome 6 Project, but the data from the 25 case reports were often incomplete, lacking especially information about milestones and behaviour. For example, most of the children in our parent cohort were described as being social, whereas this information was often not available for the literature cases.

The main aim of the Chromosome 6 Project is to create helpful information for parents and doctors. Here we report the genotypes and phenotypes of 45 individuals with proximal $6 \mathrm{q}$ deletions, the largest cohort to date. However, larger numbers of cases are needed to make more detailed and reliable descriptions of the expected phenotypes and their variability, while follow-up data will give more insight into their development and future perspectives. The Chromosome 6 Project uses social media to collect information, not only on the phenotypes and genotypes but also on what topics are important for parents and how we can better involve them in our research. The project currently has $>700$ followers on Twitter and reaches $>900$ members of the Facebook group. Unfortunately, we do not know how many of these individuals have a chromosome 6 aberration.

So far, little attention has been given to the barriers that families encounter in participating in studies like the Chromosome 6 Project, while studies requested and initiated by parents are often clinically relevant and result in highly motivated participation. Of course, there are questions about the reliability of information retrieved directly from parents. For example, one might assume that parents tend to overestimate their child's developmental level. Therefore, we only used the results of standardised developmental tests or well-defined milestones to categorise the individuals in subgroups of developmental delay. We also asked for formal behavioural diagnoses of autism spectrum disorder and ADHD, rather than relying on parents' perspectives of their child's behaviour. We are in the process of validating our online questionnaire by comparing parental data with information collected from the medical professionals. The results so far are encouraging.

\section{Concluding remarks}

Not surprisingly, the present study showed that our questionnaire led to extra and more detailed information than that available in case reports. We demonstrate that patient/ parent involvement via social media is a successful strategy 
and it will result in a growing wealth of information that will prove extremely important to parents of young children with a rare chromosome aberration. Our goal is to construct a detailed phenotype-genotype map for the complete chromosome 6 that can be used for counselling and the clinical management of patients in the future.

\section{URLS}

\author{
https://decipher.sanger.ac.uk/ \\ http://genome.ucsc.edu/ \\ http://exac.broadinstitute.org \\ https://www.chromosome6.org/ \\ http://www.ecaruca.net \\ https://www.omim.org/
}

Acknowledgements We are grateful to all the children and families for their participation. We also thank Jackie Senior for editing the manuscript, the members of the Genomic Coordination Centre, in special Mariska Slofstra, and all the members of the Chromosome 6 Project's advisory board (Sarah Wynn, Klaas Dolsma, Mieke van Leeuwen, Marianne Doornbos, Nicole de Leeuw and Agnies van Eeghen) for their input. Our special thanks go to our contact for the Chromosome 6 Facebook group, Pauline Bouman. This work was supported by a grant from ZonMw (113312101) and by crowd-funding organised by chromosome 6 parents.

\section{Compliance with ethical standards}

Conflict of interest The authors declare that they have no conflict of interest.

Open Access This article is licensed under a Creative Commons Attribution 4.0 International License, which permits use, sharing, adaptation, distribution and reproduction in any medium or format, as long as you give appropriate credit to the original author(s) and the source, provide a link to the Creative Commons license, and indicate if changes were made. The images or other third party material in this article are included in the article's Creative Commons license, unless indicated otherwise in a credit line to the material. If material is not included in the article's Creative Commons license and your intended use is not permitted by statutory regulation or exceeds the permitted use, you will need to obtain permission directly from the copyright holder. To view a copy of this license, visit http://creativecommons. org/licenses/by/4.0/.

\section{References}

1. Hopkin RJ, Schorry E, Bofinger M, et al. New insights into the phenotypes of 6q deletions. Am J Med Genet. 1997;70:377-86.

2. Feenstra I, Fang J, Koolen DA, et al. European Cytogeneticists Association Register of Unbalanced Chromosome Aberrations (ECARUCA); an online database for rare chromosome abnormalities. Eur J Med Genet. 2006;49:279-91.

3. Farmer AD, Bruckner Holt CEM, Cook MJ, Hearing SD. Social networking sites: a novel portal for communication. Postgrad Med J. 2009;85:455-9.

4. Swertz MA, Dijkstra M, Adamusiak T, et al. The MOLGENIS toolkit: rapid prototyping of biosoftware at the push of a button. BMC Bioinformatics. 2010;11(Suppl 12):S12.
5. Huang N, Lee I, Marcotte EM, Hurles ME. Characterising and predicting haploinsufficiency in the human genome. PLoS Genet. 2010;6:e1001154.

6. Lek M, Karczewski KJ, Minikel EV, et al. Analysis of proteincoding genetic variation in 60,706 humans. Nature. 2016;536:285-91.

7. Quintela I, Fernandez-Prieto M, Gomez-Guerrero L, et al. A 6q14.1-q15 microdeletion in a male patient with severe autistic disorder, lack of oral language, and dysmorphic features with concomitant presence of a maternally inherited Xp22.31 copy number gain. Clin Case Rep. 2015;3:415-23.

8. Dworschak GC, Draaken M, Hilger AC, et al. Genome-wide mapping of copy number variations in patients with both anorectal malformations and central nervous system abnormalities. Birth Defects Res Part A. 2015;103:235-42.

9. Van Esch H, Rosser EM, Janssens S, Van Ingelghem I, Loeys B, Menten B. Developmental delay and connective tissue disorder in four patients sharing a common microdeletion at 6q13-14. J Med Genet. 2010;47:717-20.

10. Vlckova M, Trkova M, Zemanova Z, et al. Mechanism and genotype-phenotype correlation of two proximal $6 \mathrm{q}$ deletions characterized using mBAND, FISH, Array CGH, and DNA sequencing. Cytogenet Genome Res. 2012;136:15-20.

11. Wang J, Dang L, Lomax B, et al. Molecular breakpoint mapping of 6q11-q14 interstitial deletions in seven patients. Am J Med Genet Part A. 2009;149A:372-9.

12. Woo K, Kim J, Kim K, et al. A de novo proximal $6 q$ deletion confirmed by array comparative genomic hybridization. Korean $\mathrm{J}$ Lab Med. 2010;30:84-8.

13. Wentzel C, Lynch SA, Stattin EL, Sharkey FH, Anneren G, Thuresson AC. Interstitial deletions at 6q14.1-q15 associated with obesity, developmental delay and a distinct clinical phenotype. Mol Syndromol. 2010;1:75-81.

14. Duarte C, Farinha RR, Santos AR, Dias P, Sousa AB, Pereira AM. Description of a child with a 6q14.1-q16.1 interstitial deletion: a very rare entity with airway manifestations. Int J Pediatr Otorhinolaryngol. 2016;84:147-50.

15. Lespinasse J, Gimelli S, Bena F, Antonarakis SE, Ansermet F, Paoloni-Giacobino A. Characterization of an interstitial deletion 6q13-q14.1 in a female with mild mental retardation, language delay and minor dysmorphisms. Eur $\mathrm{J}$ Med Genet. 2009;52:49-52.

16. Wentzel C, Anneren G, Thuresson AC. A maternal de novo nonreciprocal translocation results in a 6q13-q16 deletion in one offspring and a 6q13-q16 duplication in another. Eur J Med Genet. 2014;57:259-63.

17. Lowry RB, Chernos JE, Connelly MS, Wyse JP. Interstitial deletions at $6 \mathrm{q} 14.1 \mathrm{q} 15$ associated with developmental delay and a marfanoid phenotype. Mol Syndromol. 2013;4:280-4.

18. Pinto D, Delaby E, Merico D, et al. Convergence of genes and cellular pathways dysregulated in autism spectrum disorders. Am J Hum Genet. 2014;94:677-94.

19. Becker K, Di Donato N, Holder-Espinasse M, et al. De novo microdeletions of chromosome 6q14.1-q14.3 and 6q12.1-q14.1 in two patients with intellectual disability - further delineation of the $6 q 14$ microdeletion syndrome and review of the literature. Eur J Med Genet. 2012;55:490-7.

20. Lehman A, Thouta S, Mancini GMS, et al. Loss-of-function and gain-of-function mutations in KCNQ5 cause intellectual disability or epileptic encephalopathy. Am J Human Genet. 2017;101:65-74.

21. Webster E, Cho MT, Alexander N, et al. De novo PHIP-predicted deleterious variants are associated with developmental delay, intellectual disability, obesity, and dysmorphic features. Cold Spring Harb Mol Case Stud. 2016;2:a001172. 
22. de Ligt J, Willemsen MH, van Bon BW, et al. Diagnostic exome sequencing in persons with severe intellectual disability. N Engl J Med. 2012;367:1921-9.

23. Lelieveld SH, Reijnders MR, Pfundt R, et al. Meta-analysis of 2104 trios provides support for 10 new genes for intellectual disability. Nat Neurosci. 2016;19:1194-6.

24. Bosch PJ, Fuller LC, Sleeth CM, Weiner JA. Akirin2 is essential for the formation of the cerebral cortex. Neural Dev. 2016;11:21.

25. Park E, Kim Y, Noh H, Lee H, Yoo S, Park S. EphA/ephrin-A signaling is critically involved in region-specific apoptosis during early brain development. Cell Death Differ. 2013;20:169-80.

26. Michaelides M, Holder GE, Hunt DM, Fitzke FW, Bird AC, Moore AT. A detailed study of the phenotype of an autosomal dominant cone-rod dystrophy (CORD7) associated with mutation in the gene for RIM1. Br J Ophthalmol. 2005;89:198-206.

27. Dong S, Walker MF, Carriero NJ, et al. De novo insertions and deletions of predominantly paternal origin are associated with autism spectrum disorder. Cell Rep. 2014;9:16-23.
28. Krumm N, Turner TN, Baker C, et al. Excess of rare, inherited truncating mutations in autism. Nat Genet. 2015;47:582-8.

29. Wang T, Guo H, Xiong B, et al. De novo genic mutations among a Chinese autism spectrum disorder cohort. Nat Commun. 2016;7:13316.

30. McAllister G, Charlesworth A, Snodin C, et al. Molecular cloning of a serotonin receptor from human brain (5HT1E): a fifth 5HT1-like subtype. Proc Natl Acad Sci USA. 1992;89: $5517-21$

31. Orabona GM, Griesi-Oliveira K, Vadasz E, et al. HTR1B and HTR2C in autism spectrum disorders in Brazilian families. Brain Res. 2009;1250:14-9.

32. Le Goff C, Rogers C, Le Goff W, et al. Heterozygous mutations in MAP3K7, encoding TGF-beta-activated kinase 1, cause cardiospondylocarpofacial syndrome. Am J Hum Genet. 2016;99:407-13.

33. Ma L, Li J, Liu Y, Pang S, Huang W, Yan B. Novel and functional variants within the TBX18 gene promoter in ventricular septal defects. Mol Cell Biochem. 2013;382:121-6. 